\title{
Évaluation du Coefficient De Frottement Dans Le Cas D’un Écoulement À Surface Libre
}

\author{
Lahmar Karim \\ Jean-Pierre Larue \\ Université de Monastir, Tunisie \\ Université Paris- Est, Laboratoire de géographie physique, \\ Meudon, Paris, France
}

doi: 10.19044/esj.2016.v12n15p418 URL:http://dx.doi.org/10.19044/esj.2016.v12n15p418

\begin{abstract}
A large number of empirical formulas such as Bazin and Manning's formulas are used to estimate stream flows for design purpose. Discharge is calculated by standard equations that are all based on an empirical coefficient found to be a characteristic of the wall and not of its relative roughness features. This, in turn, would yield different results displayed during the calculation of Chezy coefficient while using standard materials. In open channel flows, features on the wall may have an important effect in assessing flow properties such as friction, velocity, and discharge. In this context, we have led an experimental study on a rectangular channel using the boundary layer and turbulence theory. The rectangular channel understudy is characterized by Plexiglas walls having three different roughness conditions. The study is carried out using abrasive papers with different dimensions. The results of the experiment reveals that for the three wall conditions (i.e. Plexiglas P220 and P80) and in the case of hydraulically smooth and torrential flow, the friction is estimated by Pranldt Von Karman's formula. The same conclusion can be drawn for the different experimental tests performed on a bi-dimensional channel with a bottom made of PVC in a hydraulically smooth and fluvial flow. However, considerable discrepancy between results obtained has been inferred. Actually, there is a difference between values calculated by Chezy coefficient formula and those calculated by Manning's formula. The latter is widely used in recent investigation especially when it comes to the transition from open channel flow to pipe flow (i.e. closed-conduit flow). In the present study, in the case of transition between a hydraulically smooth flow and a rough one (detected only in the case of P80), the friction factor is represented by a form similar to that of Colebrook White.
\end{abstract}


Keywords: Smooth channel, roughness, friction, velocity, turbulence

\section{Résumé}

Les formules classiques utilisées pour le calcul du débit telles que celles de Bazin, Manning Strickler et d’autres sont toutes basées sur le choix d'un coefficient caractéristique d'une paroi et non pas de son état de rugosité, ce qui explique les différences des valeurs obtenues lors de la détermination du coefficient de Chézy, pour un même type de matériau. En revanche, l’influence de l'état de paroi, dans le cas d'un écoulement à surface libre apparaît comme un élément de décisif pour l'évaluation du frottement, de la vitesse et du débit, d'après la théorie de la couche limite et de la turbulence. Dans ce contexte nous avons mené une étude expérimentale, sur un canal rectangulaire en plexiglas avec trois états de rugosité en utilisant du papier abrasif avec des dimensions différentes, ce qui nous a permis de déduire que pour les trois états de paroi, du plexiglas, du P220 et du P80 et dans le cas d'un régime hydrauliquement lisse et torrentiel, le frottement peut être bien estimé par la formule de Pranldt Von Karman, utilisée pour les écoulements en charge. La même conclusion a été faite pour les essais que nous avons effectués sur un canal large avec un fond en PVC en régime hydrauliquement lisse et fluvial, alors qu'une divergence considérable a été constatée entre les valeurs du coefficient de Chezy calculées par cette formule et celles calculées par la formule de Manning Strickler que les auteurs utilisent dans des études récentes du passage d'un écoulement à surface libre à un écoulement en charge. Dans la zone de transition d'un écoulement hydrauliquement lisse à un écoulement rugueux, détectée pour un seul cas de rugosité, le cas du P80, le coefficient de frottement est représenté par une relation de forme semblable à celle de Colebrook White.

Mots clés: Canal lisse, rugosité, frottement, vitesse, turbulence

\section{INTRODUCTION}

Depuis le dix-huitième siècle, le frottement dans les canaux découverts a été représenté par le coefficient de CHÉZY, exprimé par des formules diverses basées sur le choix du coefficient caractéristique d'un état de rugosité ; qui est souvent très délicat à fixer; vu la disproportion existent entre le nombre de coefficients proposé et la variété considérable des types de parois.

L’étude d’un écoulement partiellement à surface libre et en charge nécessite l'évaluation du frottement, de la façon la plus précise possible, en fonction du type d'écoulement. 
Des études menées récemment, [B. Trajkovic et al, 1999], [M. Gomez et V. Achiaga, 2001, [J. Vasconcelos et S. Wright, 2004] et [C. Bourdarias et S. Gerbi, 2006], utilisent le coefficient de Manning pour exprimer le frottement.

Ceci peut induire des erreurs appréciables, d'où l'idée d'appliquer la méthodologie suivie en écoulement en charge dans le but d'uniformiser la formulation pour les deux types d'écoulement cela a été tentée par Keulegan (J. Cousteix, 1989) et poursuivi par les recherches systématiques entreprises par Zegjda, Graf (W. Graf et M. Altinaker, 1993) et Wang (Wang. J, 1991).

Dans ce cadre nous avons jugé utile de faire une étude expérimentale s'intéressant à la détermination du coefficient de frottement, f, dans deux types de canaux.

\section{Aperçu bibliographique}

En 1775, CHÉZY a établi l'expression de la vitesse; dans le cas d'un écoulement uniforme (E.Crausse, 1951) :

$$
U=C\left(R_{h}\right)^{1 / 2}
$$

Avec:

$U$ : vitesse moyenne de l'écoulement $(\mathrm{m} / \mathrm{s})$

$C:$ coefficient de Chezy $\quad\left(\mathrm{m}^{1 / 2} / \mathrm{s}\right)$

$R h$ : rayon hydraulique $\quad(\mathrm{m})$

Une variété de formules, exprimant le coefficient (C) dit de CHÉZY ont ensuite été proposées. Nous retiendrons :

La formule de BAZIN (E.Crausse, 1951) :

$$
C=87 /\left[1+\left(\gamma /\left(R_{h}\right)^{1 / 2}\right]\right.
$$

La formule de Ganguillet Kutter (E.Crausse, 1951) :

$\mathrm{C}=[23+(0,000155 / \mathrm{n})+(1 / \mathrm{n})] /[1+\mathrm{n}(23+(0,000155 / \mathrm{n})]$

La formule de MANNING (E.Crausse, 1951) :

$$
\mathrm{C}=(1 / \mathrm{n})(\mathrm{Rh})^{1 / 6}
$$

Avec :

$U$ : vitesse moyenne de l'écoulement $(\mathrm{m} / \mathrm{s})$

$C:$ coefficient de Chezy $\quad\left(\mathrm{m}^{1 / 2} / \mathrm{s}\right)$

$R h$ : rayon hydraulique

(m)

$I$ : pente du fond

$\gamma:$ Coefficient de BAZIN

$n$ : coefficient de Manning

Les études basées sur la théorie des écoulements sous pression entreprises par certains auteurs [W.Graf, 1990 et J. Wang ,1991] proposent pour la répartition de la vitesse : 


\section{Ecoulement hydrauliquement lisse}

$U / U_{f}=(1 / k) \operatorname{Ln}\left[\left(R_{h} U_{f}\right) / v\right]+\beta$

Keulegan (W.Graf, 1990), a pris pour la valeur de, $\beta$ la moyenne obtenue par plusieurs expériences et qui est égale à 3,5. Par contre Graf, donne un coefficient de 5,1.

\section{Zone de transition}

$$
\begin{aligned}
& (1 / \sqrt{f})=-2 \log \left[\frac{\mathrm{k} / \mathrm{Rh}}{a_{f}}+\frac{b_{f}}{R_{e} \sqrt{f}}\right] \\
& \text { Avec }: 12<a_{f}<15 \text { et } 0<b_{f}<6
\end{aligned}
$$

\section{DÉTERMINATION DU COEFFICIENT DE FROTTEMENT Définition}

C'est le rapport de la contrainte tangentielle de cisaillement $\left(\tau_{p}\right)$ à la paroi avec l'énergie cinétique. Il est donné par J.Cousteix (1989) :

$$
\begin{aligned}
& C_{f}=\tau_{p} /\left(U^{2} / 2\right) \\
\text { Avec : } & \tau_{p}=\rho U_{f}^{2} \\
\text { et } & U_{f}=\left(g R_{h} j\right)^{1 / 2}
\end{aligned}
$$

En pratique $\left(\mathrm{C}_{f}\right)$ est remplacé par un autre coefficient (f) dit de perte de charge, ou de frottement :

$$
\begin{array}{cl}
C_{f}=f / 4 \\
\text { D'ou } & \left(U / U_{f}\right)=(8 / f)^{1 / 2}
\end{array}
$$

Avec:

$U_{f}$ : vitesse de frottement $(\mathrm{m} / \mathrm{s})$

$f$ : coefficient de frottement "perte de charge »

$C_{f}$ : coefficient de frottement

$j$ : pente de la ligne d'eau

$\tau_{p}$ : Contrainte tangentielle à la paroi $\left(\mathrm{kg} \mathrm{m}^{-1} / \mathrm{s}^{2}\right)$

$g:$ accélération de la pesanteur $\left(\mathrm{m} / \mathrm{s}^{2}\right)$

\section{Régime hydrauliquement lisse}

Ce régime est observé dans le cas où nous avons : $0<\operatorname{Ref}<5$

Sachant que Ref est donné par :

$$
\operatorname{Ref}=\left(k U_{f}\right) / v
$$

Il est appelé nombre de Reynolds de frottement.

Dans ce cas le coefficient de frottement est fonction du nombre de Reynolds (Re).

3,3 Régime de transition. 
Lors de la transition d’un régime hydrauliquement lisse à un régime complètement rugueux, nous avons : $5<$ Ref $<70$.

Dans ce cas les effets de la viscosité et de la rugosité interviennent simultanément.

\section{ÉTUDE EXPÉRIMENTALE}

Les séries d'essais ont été effectués sur deux installations expérimentales.

La première est composée d'un réservoir d'alimentation en eau, d'un canal d'essai de forme rectangulaire de $4 \mathrm{~m}$ de longueur, de $15 \mathrm{~cm}$ de hauteur et de $14 \mathrm{~cm}$ de largeur, et d'un bac de récupération muni d'un déversoir triangulaire permettant la mesure du débit. La hauteur d'eau est lue sur des tubes piézométriques.

Trois états de rugosité ont été utilisés : $1^{\mathrm{er}}$ état :

Le fond et les parois latérales sont en plexi- glas, la rugosité absolue de ce dernier est de : $\mathrm{k}=0.0006 \mathrm{~cm}$ $2^{\text {ème état : }}$

Le fond et les parois latérales du canal sont rugueux, du papier abrasif a été utilisé, constitué de macro grains d'oxyde d'Aluminium et désigné par P220. ( $\mathrm{k}=0,01 \mathrm{~cm})$ Voir figure.1

Deux méthodes ont été utilisées pour la mesure de la dimension de la rugosité absolue. Indirectement en utilisant la courbe granulométrique du papier abrasif pour déterminer le diamètre dont $50 \%$ des grains ont des diamètres plus fins et directement en utilisant un rugosimètre type Hobson Taylor pour mesurer la hauteur moyenne des aspérités. $3^{\text {ème }}$ état :

Le fond et les parois latérales du canal sont rugueux, du papier abrasif a été utilisé, constitué de macro grains d'oxyde d'Aluminium et désigné par P80. ( $\mathrm{k}=0,02 \mathrm{~cm})$ voir figure. 2

La dimension de la rugosité absolue a été mesurée de la même manière que précédemment. 


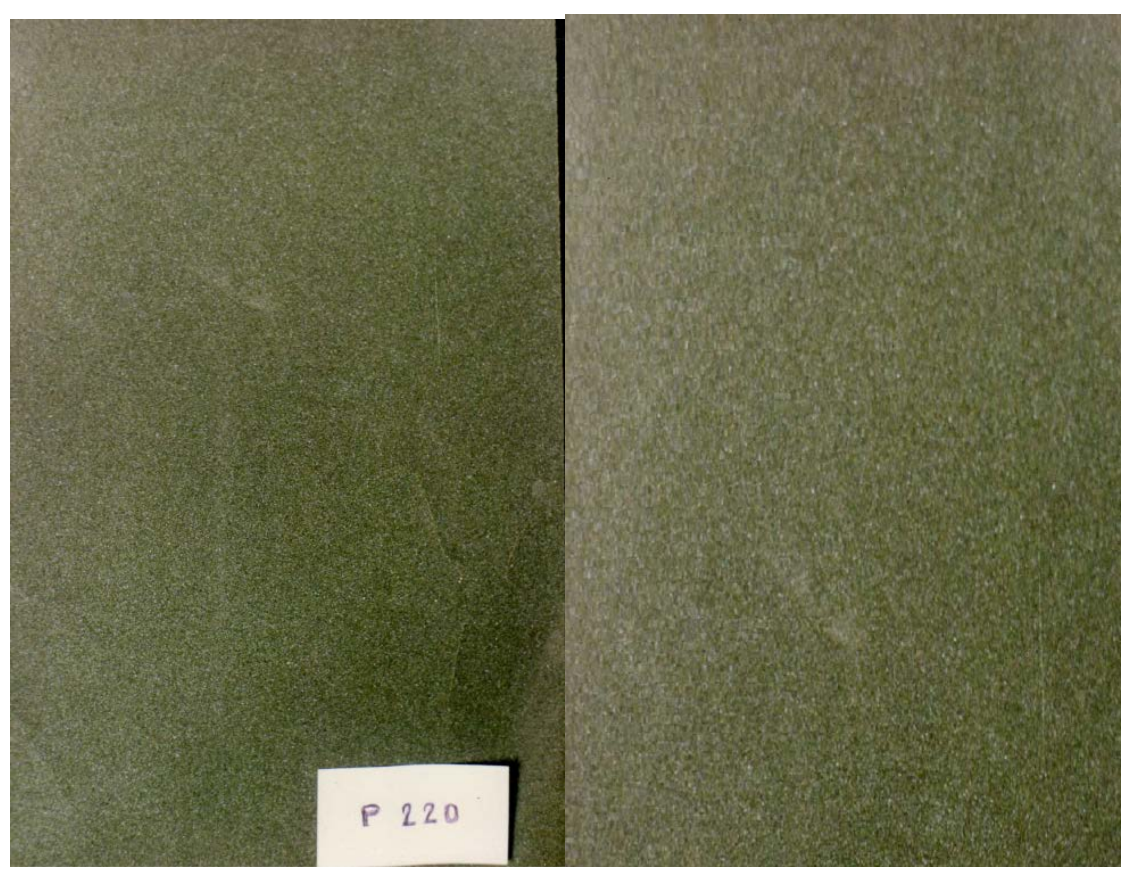

Fiqure 1 : Papier abrasif « P220»

Figure 2: Papier abrasif «P80»

Les débits sont mesurés en aval du canal, à l'aide d'un déversoir triangulaire, ayant une hauteur de pèle de $18 \mathrm{~cm}$ posé, sur le bac de récupération .le débit est calculé par la formule de B.Bahidj (1988) : $Q=0,0172611 h_{d}^{2,35}$

Où $h_{d}$, est la hauteur déversée au dessus du seuil du déversoir en mm, mesurée au moyen d'un limnimètre et $Q$ le débit en $1 / \mathrm{s}$.

La deuxième installation comprend un canal de forme rectangulaire de 2,35 m de longueur, de $15 \mathrm{~cm}$ de hauteur et de $61,2 \mathrm{~cm}$ de largeur. Il est alimenté au moyen d'une pompe.les essais y sont effectués de sorte que le rapport de la largeur du canal à la profondeur d'eau dépasse la valeur, 5 , a fin de répondre à la condition d'un canal bidimensionnel large ou l'influence de la rugosité du fond est prépondérante.

Pour cet article nous examinons trois cas de rugosité : $1^{\mathrm{er}}$ Etat :

Une forme lisse du fond constituée d'une plaque en PVC dure, qui donne une dimension moyenne caractéristique de la rugosité de $0,0065 \mathrm{~cm}$. $2^{\mathrm{em}}$ Etat :

Une forme ondulatoire en plastique dont les vides sont remplis avec du plâtre présente une caractéristique de $0,9 \mathrm{~cm}$. $3^{\text {ème }}$ état :

Des cailloux de forme non uniforme, dont la hauteur est comprise entre $0,5 \mathrm{~cm}$ et $1,9 \mathrm{~cm}$ avec une valeur moyenne de $1,28 \mathrm{~cm}$ 
Le débit refoulé est mesuré grâce à un venturimètre par la lecture de la différence de pression lue sur un manomètre différentiel, provoquée par le rétrécissement. L'étalonnage de ce débitmètre a permis d'établir l'expression suivante :

$$
Q=0,237132(\Delta h)^{0,5}
$$

Avec $\Delta h$ la différence pression en mm

\section{Hypothèses de calcul}

Nous calculons la vitesse moyenne de l'écoulement $U$, la vitesse de frottement $U_{f}$, le nombre de Reynolds $R_{e}$, le nombre de Reynolds de frottement $R_{e f}$, le rayon hydraulique $R_{h}$ et le coefficient de frottement $f$ en considérant les hypothèses suivantes.

- L'écoulement est permanent.

- $\quad$ Le fluide est incompressible

- $\quad$ Le transport des solides est négligeable.

- La distribution de la pression est considérée comme hydrostatique.

\section{Résultats expérimentaux et interprétation}

Pour la première installation nous distinguons les trois cas suivants:

\section{Canal rectangulaire en plexi -glas}

$$
\text { Pour : } 5,10^{4}<R_{e}<9,10^{5}
$$

L'écoulement est torrentiel et hydrauliquement lisse. La figure 3 représente la variation du rapport, $\frac{U}{U_{f}}$ en fonction de y. Avec $y=\operatorname{Ln} \frac{R_{h} U_{f}}{v}$

L'application d'un ajustement linéaire donne la relation suivante :

$$
\frac{U}{U_{f}}=2,6 \operatorname{Ln} \frac{R_{h} U_{f}}{v}+5,11
$$

L'évolution du coefficient de frottement en fonction du nombre de Reynolds suit une loi puissance, donnée par : $f=0,162 R_{e}^{-0,207}$

\section{Canal rectangulaire en $P 220$}

Pour : $6,10^{4}<R_{e}<9,510^{5}$

L'écoulement est torrentiel et hydrauliquement lisse. La figure.4 représente la variation de la distribution de vitesse en fonction de $y=\operatorname{Ln} \frac{R_{h} U_{f}}{v}$

l'allure de la courbe est une droite dont l'équation est obtenue par l'application d'un ajustement linéaire : 


$$
\frac{U}{U_{f}}=2,61 \operatorname{Ln} \frac{R_{h} U_{f}}{v}+4,97
$$

En considérant la variation du nombre de Reynolds, le coefficient de frottement suit la loi :

$$
f=0,162 R_{e}^{-0,201}
$$

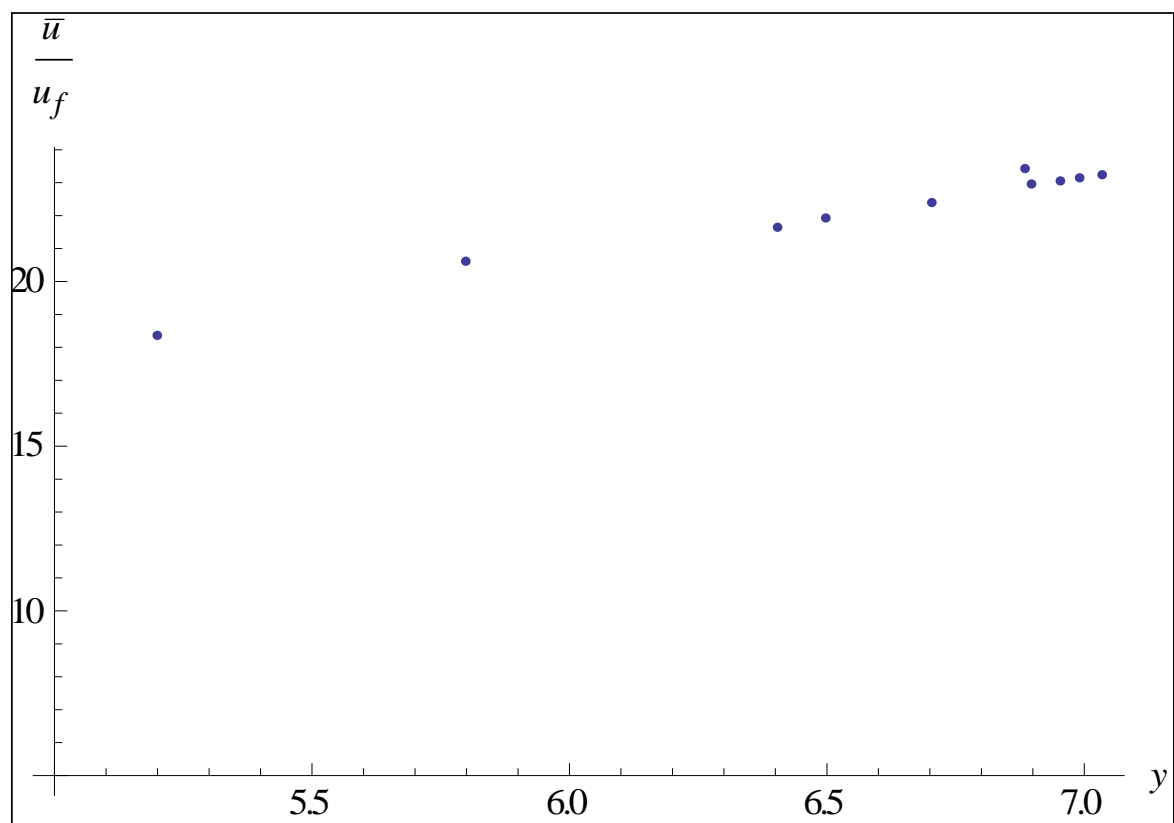

$\underline{\text { Fiqure } 3}$ : Évolution de la distribution de la vitesse pour le plexiglas.

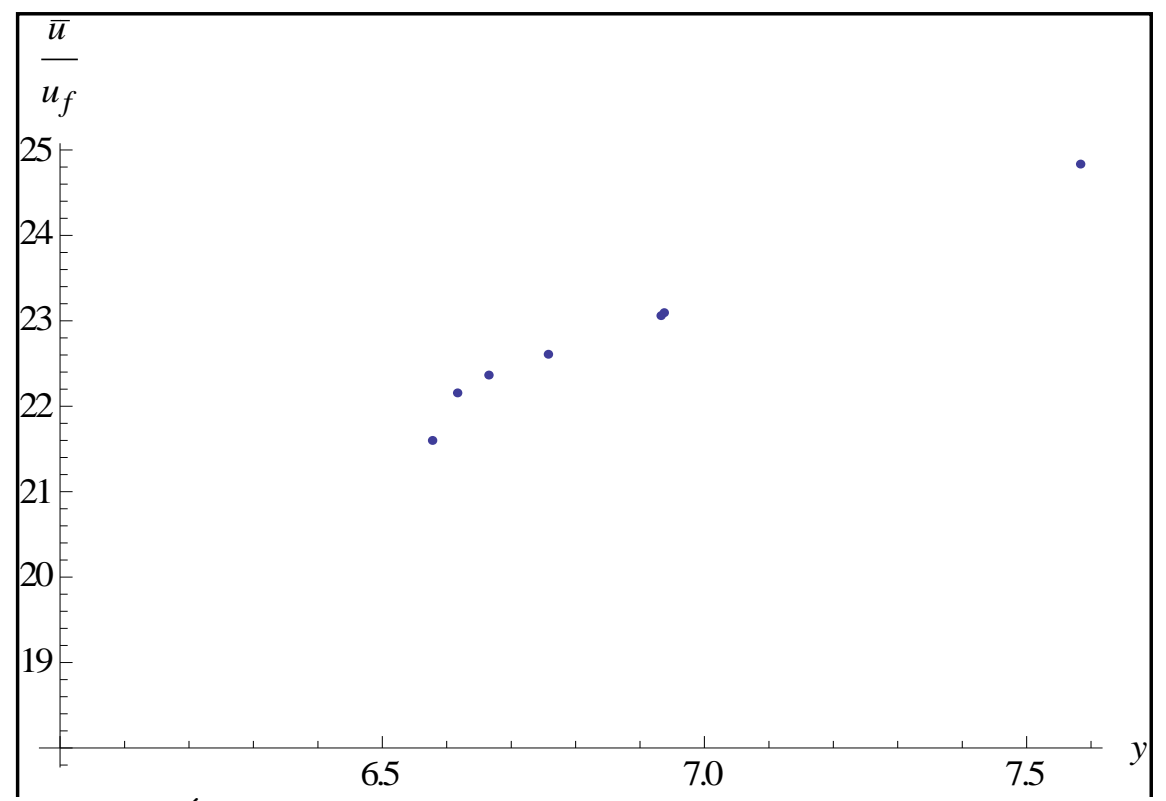

Fiqure 4: Évolution de la distribution de la vitesse pour le papier abrasif P220 


\section{Canal rectangulaire en $\mathbf{P 8 0}$}

Pour : $2,104<\operatorname{Re}<3,105$

L'écoulement est en grande partie torrentiel .Deux zones d'écoulement sont distingués :

Zone hydrauliquement lisse :

Dans cette zone les résultats expérimentaux montrent que la distribution de la vitesse suit une loi linéaire en fonction de $y=\operatorname{Ln} \frac{R_{h} U_{f}}{v}$ .Nous avons :

$\frac{U}{U_{f}}=2,59 \operatorname{Ln} \frac{R_{h} U_{f}}{v}+5,96$

Les points expérimentaux s'alignent sur une droite ayant pour équation aussi :

$f=0,162 R_{e}^{-0,201}$

\section{Zone de transition}

D’après la figure.5, nous constatons que le coefficient de frottement décroît au fur et à mesure que $R_{e}$ augmente avec le poli relatif $\frac{R_{h}}{k}$. Pour ce régime $f$ dépend de $R_{e}$ et de $\frac{R_{h}}{k}$ simultanément. Le comportement du coefficient de frottement est régi par l'expression (6), ci avant et les coefficients $\mathrm{a}_{f}$ et $\mathrm{b}_{f}$ sont déterminés par l'application de la méthode des moindre carrés à deux variables :

Soit la fonction d'approximation

$$
(1 / \sqrt{f})=-2 \log \left[\frac{\mathrm{k} / \mathrm{Rh}}{a_{f}}+\frac{b_{f}}{R_{e} \sqrt{f}}\right]
$$

Nous avons le triplet : $\mid \begin{aligned} & z_{i}=10^{-\frac{1}{2 \sqrt{f}}} \\ & y_{i}=\frac{1}{\operatorname{Re} \sqrt{f}} \\ & x_{i}=\frac{k}{R h}\end{aligned}$

Soit la fonction $\mathrm{g}(\mathrm{x}, \mathrm{y})$ tel que : 
$g(x, y)=\sum_{i}^{n} a_{j} v_{i}\left(x_{i}, y_{i}\right)$

$i=1, n$

$j=1,2$

Et soit E l'erreur quadratique :

$E=\sum_{1}^{n}\left[z_{i}-g\left(x_{i}, y_{i}\right)\right]^{2}$

En minimisant l'erreur, nous obtenons :

$\frac{\partial E}{\partial A}=\sum-2 x_{i} z_{i}+2 A x_{i}^{2}+2 B x_{i} y_{i}$

$\frac{\partial E}{\partial B}=\sum-2 y_{i} z_{i}+2 A x_{i} y_{i}+2 B y_{i}^{2}$

Soit le système à résoudre :

$\left[\begin{array}{cc}\sum x_{i}^{2} & \sum x_{i} y_{i} \\ \sum x_{i} y_{i} & \sum y_{i}^{2}\end{array}\right]\left[\begin{array}{l}A \\ B\end{array}\right]=\left[\begin{array}{l}\sum x_{i} z_{i} \\ \sum y_{i} z_{i}\end{array}\right]$

avec:

$A=\frac{1}{a_{f}}$

$B=\frac{1}{b_{f}}$

Nous trouvons :

$\mathrm{a}_{f}=14,1$ et $\mathrm{b}_{f}=0,725$

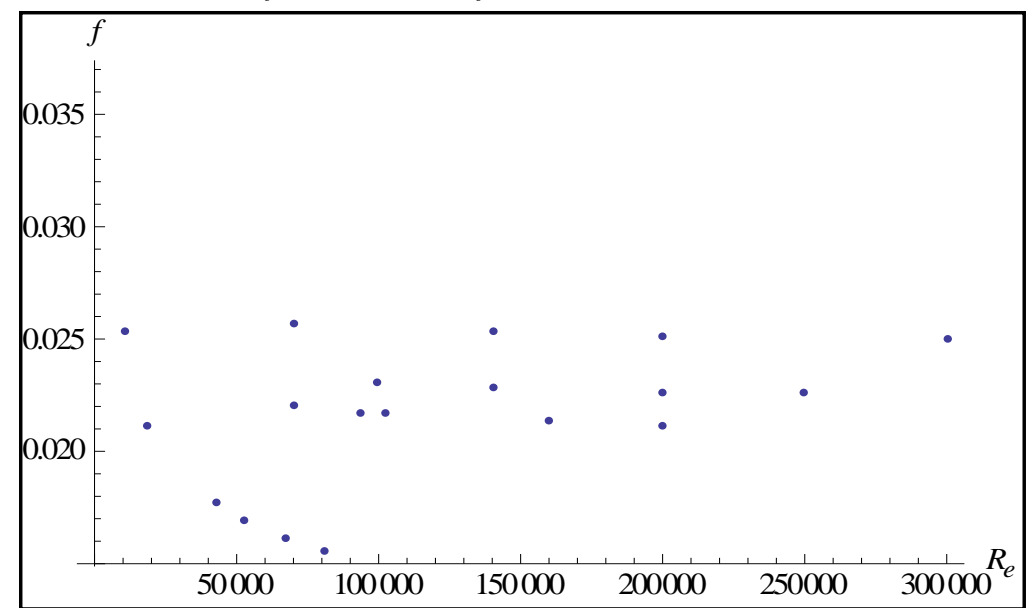

Fiqure 5: Évolution du coefficient de frottement en fonction de Re pour le papier abrasif P80

Pour la deuxième installation un seul cas de rugosité a été considéré. 


\section{Canal bidimensionnel à fond en PVC}

Pour : $2.10^{4}<R_{e}<3.10^{5}$

L'écoulement est fluvial et hydrauliquement lisse. La figure 6 montre que les points expérimentaux s'alignent sur une droite dont l'équation est obtenue par ajustement linéaire :

$\frac{U}{U_{f}}=2,71 \operatorname{Ln} \frac{R_{h} U_{f}}{v}+3,72$

Le coefficient de frottement $f$ évolue dans ce cas en fonction du nombre de Reynolds selon la loi :

$f=0,337 R_{e}^{-0,27}$

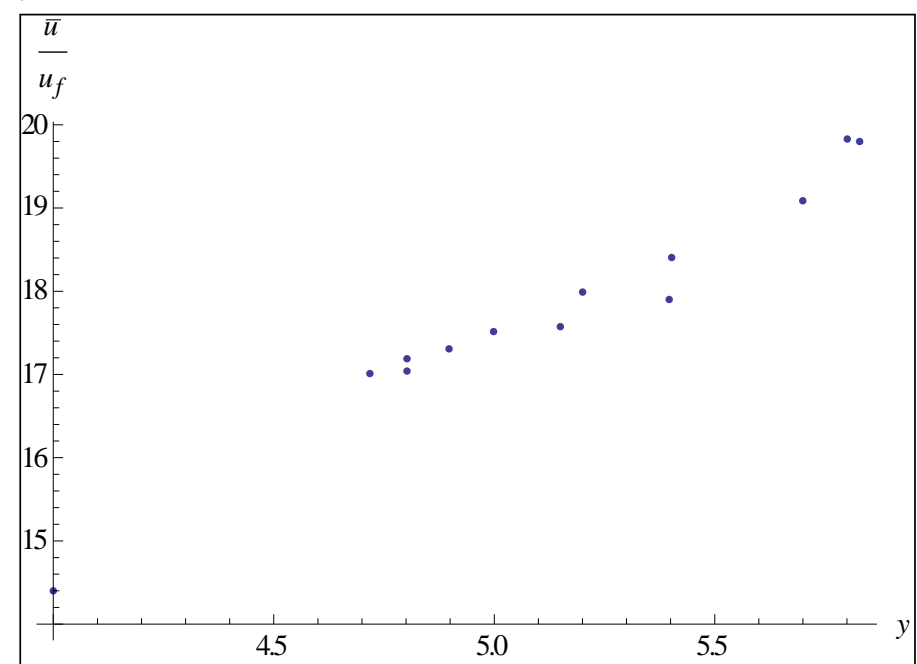

Fiqure 6: Évolution de la distribution de la vitesse pour le PVC

\section{Canal bidimensionnel, à fond, à rugosité ondulatoire}

La variation du coefficient de frottement $\mathrm{f}$ en fonction du nombre de Reynolds (Re) et pour différents polis relatifs $\left(\frac{R_{h}}{k}\right)$ est donnée dans la figure .7. Cette dernière montre qu'il existe deux zones distinctes d'écoulement et que les points expérimentaux se situent sur des courbes parallèles, dont chacune correspond à une valeur du poli relatif différente.

\section{Zone complètement rugueuse}

\section{Pour : $8,10^{3}<\mathrm{R}_{\mathrm{e}}$ et $\mathrm{F}_{\mathrm{r}}<1$}

La figure 8 indique que $\mathrm{f}$ est représenté par des courbes parallèles pouvant être assimilées à des droites .Cette zone est d'autant plus grande que la valeur du poli est faible. Le coefficient de frottement $\mathrm{f}$ ne dépend que de $\left(\frac{R_{h}}{k}\right)$, son comportement est régi par l'équation suivante : 


$$
\frac{1}{\sqrt{f}}=2,03 \log \frac{R_{h}}{k}+2,16
$$

\section{Zone de transition}

$$
\text { Pour : } 10^{3}<R_{e}<2,10^{3} \text { et } \quad F_{r}<1
$$

Les points de la formule de zone de transition, commencent par une croissance jusqu'à une limite de $(\mathrm{Re})$ qui devient plus importante avec l'augmentation du poli relatif. Or le coefficient de frottement dépend à la fois du nombre de Reynolds et de la valeur de $\frac{R_{h}}{k}$.

En suivant la même procédure que pour le cas du canal rectangulaire en P80 nous obtenons :

$$
(1 / \sqrt{f})=-2 \log \left[\frac{\mathrm{k} / \mathrm{Rh}}{a_{f}}+\frac{b_{f}}{R_{e} \sqrt{f}}\right]
$$

Avec :

$\mathrm{a}_{f}=12,66$ et $\mathrm{b}_{f}=1,54$

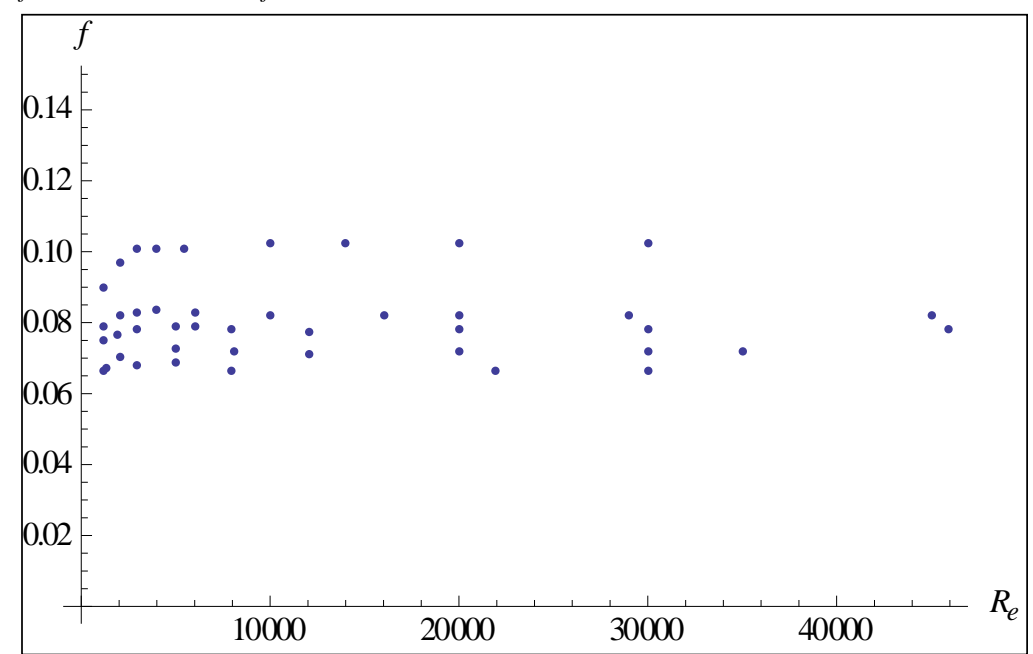

Figure 7: Évolution du coefficient de frottement en fonction de Re pour le fond, à rugosité ondulatoire

\section{Canal bidimensionnel, à fond, à rugosité à galets non uniformes}

La figure9 représente l'évolution du coefficient de frottement $f$ en fonction du nombre de Reynolds $\left(R_{e}\right)$ pour différents polis relatifs .Nous distinguons:

\section{Zone complètement rugueuse}

Pour : $6,10^{3}<R_{e}$ et $\quad F_{r}<1$

Les points expérimentaux s'alignent sur des droites parallèles. Le coefficient de frottement dépend uniquement du poli relatif. Il est régi par : 
$\frac{1}{\sqrt{f}}=1,96 \log \frac{R_{h}}{k}+1,45$

Zone de transition

Pour : Pour : $10^{3}<R_{e}<2,10^{4}$ et $\quad F_{r}<1$

Les points expérimentaux commencent par une augmentation de, $f$, suivant, $R_{e}$, et une diminution suivant, $\frac{R_{h}}{k}$.L'équation qui régit cette évolution est :

$(1 / \sqrt{f})=-2 \log \left[\frac{\mathrm{k} / \mathrm{Rh}}{a_{f}}+\frac{b_{f}}{R_{e} \sqrt{f}}\right]$

Avec :

$$
\mathrm{a}_{f}=5,5 \text { et } \mathrm{b}_{f}=1,54
$$

Le coefficient 5,5 ne concorde pas avec ceux de W .Graf (1993) .Ceci est dû à la forme des aspérités qui n’est pas régulière.

Expression du coefficient de Chézy :

En considérant la zone complètement rugueuse dans les deux cas de rugosité ci avant. Le coefficient de Chézy, C, est exprimé par deux relations différentes :

Rugosité ondulatoire $(\mathrm{k}=0,9 \mathrm{~cm})$

$C=16,2\left(\frac{R_{h}}{k}\right)^{0.258}$

Rugosité à galets non uniformes $(\mathrm{k}=1,28 \mathrm{~cm})$

$C=14,66\left(\frac{R_{h}}{k}\right)^{0.37}$

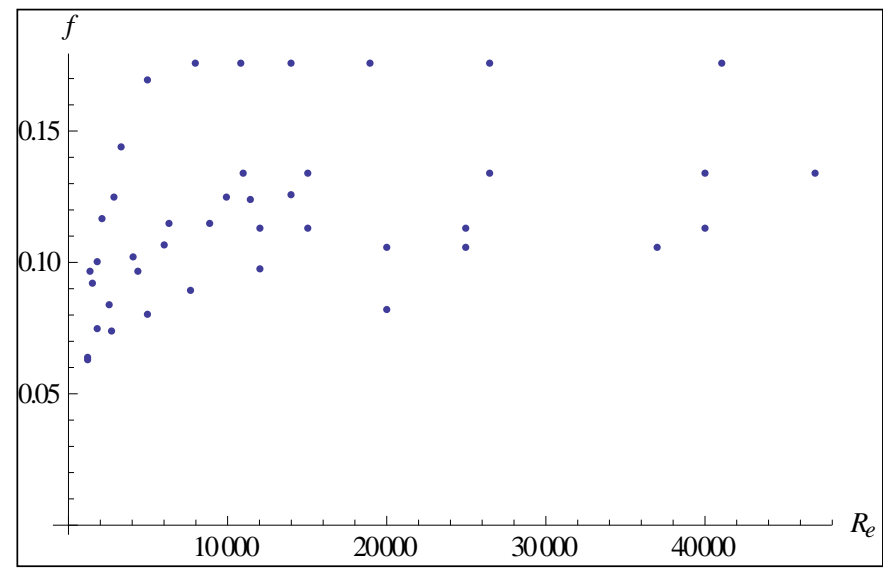

Fiqure 8: Évolution du coefficient de frottement en fonction de Re pour le fond, à rugosité à galets non uniformes 


\section{Synthèse}

Les résultats des essais expérimentaux, effectués, ont permis de constater que dans :

Le canal rectangulaire (régime hydrauliquement lisse et torrentiel) : f, se comporte suivant l'équation :

$$
\left.(8 / f)^{1 / 2}=(1 / K) \operatorname{Ln}\left(R_{h} U_{f}\right) / v\right)+B
$$

Où, $v$ est la viscosité cinématique en $\left(\mathrm{m}^{2} / \mathrm{s}\right)$

Les valeurs des constantes $\mathrm{K}$ et $\mathrm{B}$ sont déterminées par un ajustement linéaire, pour les trois cas de rugosité comme suit :

Cas du plexiglas : $\mathrm{K}=0,39$ et $\mathrm{B}=5,11$

Cas du P220 : $\mathrm{K}=0,38$ et $\mathrm{B}=4,97$

Cas du P80 : $\mathrm{K}=0,39$ et $\mathrm{B}=5,96$

De la même manière nous obtenons, pour le canal large avec un fond en PVC :

$$
\mathrm{K}=0,37 \text { et } \mathrm{B}=3,72
$$

Les différentes valeurs de $\mathrm{K}$ sont proches de la valeur universelle de Von Karman, qui est d'environ 0.4 (J. Cousteix, 1989) et pour les valeurs de B, nous remarquons qu'elles sont proches de celle trouvée par Graf (W. Graf et Altinaker, 1993) qui est de 5.1 mais elles diffèrent de celle destinée aux conduites forcées qui est de 3.5. La comparaison de ces résultats, dans les cas du plexiglas, du P220 et du P80, avec ceux obtenus, pour le cas d'un canal large, avec un fond en PVC, en régime fluvial ,montre qu'ils sont proches sauf que la valeur de B pour le canal large est proche de celle obtenue par Keulegan (J. Cousteix,1989), qui est de 3.5. La constante $B$ dépend de la forme du canal et du nombre de Froude.

La comparaison entre la formulation du type puissance obtenue dans le cas de la première installation, où l'écoulement est en grande partie torrentiel, avec la formule de Blasius, nous permet de constater un écart de $17 \%$ entre l'exposant déterminé expérimentalement et celui de Blasius. Dans le cas de la deuxième installation ou l'écoulement un régime torrentiel, il existe une déviation de $-8 \%$.

Ceci indique que la formule de Blasius n'est valable que pour de faibles valeurs du nombre de Reynolds comme il a été noté par d'autres auteurs (M. Hug, 1975).

Dans la zone de transition, qui apparaît lors des essais effectués dans le cas du papier abrasif désigné par P80, de la rugosité ondulatoire et celle à galets non uniformes le comportement du coefficient de frottement est régi par l'expression (5), ci avant, avec :

Pour le papier abrasif P80 : $\quad \mathrm{a}_{f}=14,1$ et $\mathrm{b}_{f}=0,725$

Pour la rugosité ondulatoire : $\mathrm{a}_{f}=12,66$ et $\mathrm{b}_{f}=1,54$ 
Pour les galets non uniformes : $\mathrm{a}_{f}=5,5$ et $\mathrm{b}_{f}=1,54$

Le coefficient de frottement se comporte, avec une différence dans les coefficients de la formulation. Il est obtenu par la combinaison de la formule destinée à l'écoulement hydrauliquement lisse et celle destinée à l'écoulement complètement rugueux.

Dans la zone complètement rugueuse apparaissant pour les cas de rugosités ondulatoires et à galets non uniformes, $\mathrm{f}$, dépend uniquement du poli relatif. Il se comporte suivant une loi de forme similaire à celle de Nikuradsé.

La représentation graphique du coefficient de CHÉZY exprimé par la formule de Manning Strickler, en considérant que le coefficient de CHÉZY égal à 0,013 , et par la formule déduite de la théorie des écoulements en charge, $C=(8 g / f)^{1 / 2}$, pour une paroi en plexiglas, montrée dans la figure7, cidessous, permet de constater une large différence entre les résultats pour le cas d'un écoulement à surface libre hydrauliquement lisse et torrentiel.

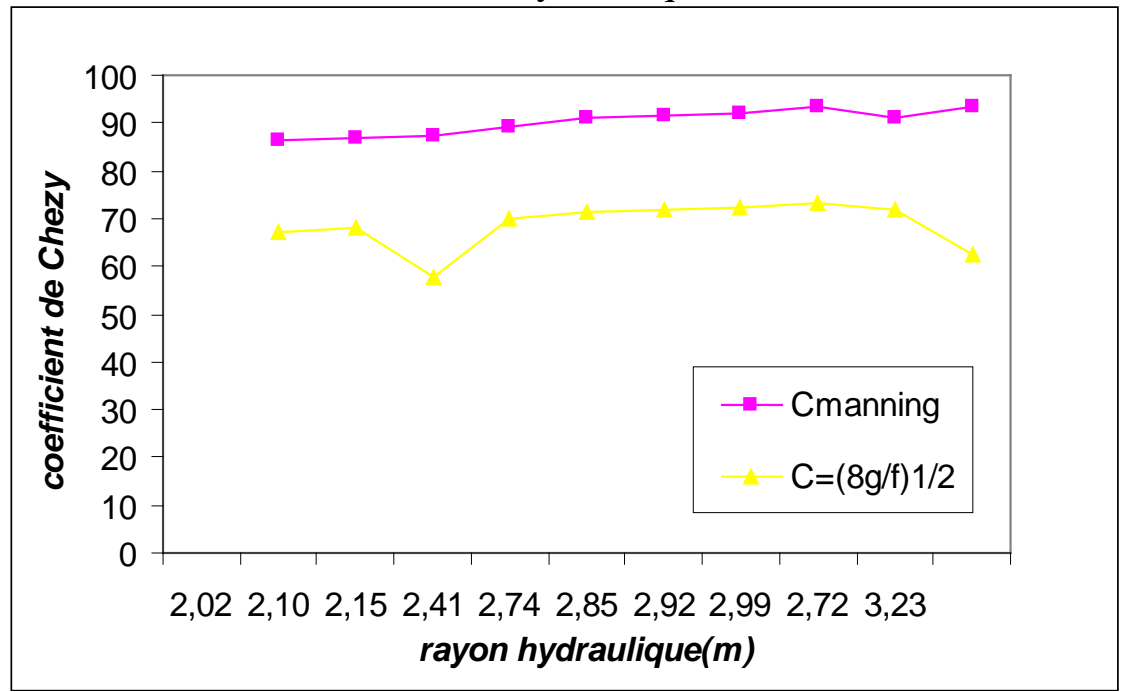

Figure 9 : Différence entre un écoulement à surface libre lisse et torrentiel.

\section{Conclusion}

Les formules anciennes de Bazin, de Kutter et de Manning, exprimant le coefficient de Chezy, à travers duquel est représenté le frottement dans les canaux peuvent induire à une sous estimation ou surestimation du débit et influencer la détection de la zone de mise en charge d'un collecteur d'assainissement ou d'une galerie hydroélectrique étant initialement à surface libre.

Notre étude expérimentale a permis de constater que le coefficient de frottement, f, dans le cas des canaux hydrauliquement lisse se comporte suivant l'équation de Pranldt Von Karman, basée sur la théorie de la 
turbulence, qui est destinée aux conduites forcées, la relation de Blasius n’est valable que pour de faibles valeurs du nombre de Reynolds et dans la zone de transition il se comporte suivant la relation universelle de ColebrookWhite, avec des coefficients différents que nous recommandons d'utiliser pour les écoulements libres .

Dans la zone complètement rugueuse le coefficient de frottement est dépendant du poli relatif par une relation similaire à celle de Nikuradsé.

Ce travail contribue à une reconsidération de l'estimation du frottement pour le cas d'un écoulement à surface libre et pour l'étude des mises en charge.

\section{References:}

TRAJKOVIC B, IVETIC M, CALOMINO F. 1999 Investigation of transition from free surface to .pressurized flow in circular pipe. Water Science.Technology.Vol39, nº: 105-112

BAHIDJ B. 1988 Contribution à l'étude des conditions de formation d'un ressaut. Thèse de Magister. Ecole Nationale Polytechnique d'Alger.

BORDARIAS C, GERBI S. 2006 Journal of computational and applied mathematics. Vol $209 \mathrm{~N}^{\circ} 1$ : 109-131

CRAUSSE E. 1951 Hydraulique des canaux découverts. EYROLLES.

COUSTEIX J. 1989 Turbulence et couche limite. Collection de la cheveche. CEPADUES.

VASCONCELOS J, WRIGHT S 2004 Numerical modelling of the transition between free surface and pressurized flow in stormsewers .Monograph12: Innovative modelling of urban water systems, published by CHI ${ }^{\circ} \mathrm{R} 220$ WANG J. 1991 Resistance of flow over rough bed. Journal of hydraudynamics. China ocean press.

GOMEZ M, ACHIAGA V. 2001 Mixed flow modelling produced by pressure fronts from upstream and downstream extrems.Conference proceeding paper: Urban Drainage Modeling. Published by ASCE: 401- 470. GRAF W. 1990 Uniform flow in a smooth open chanel.Journal of hydraulic research.V27 $\mathrm{n}^{\circ} 5$.

GRAF W, ALTINAKER M. 1993 Hydraulique fluviale. Tome1. Presse Polytechnique et Universitaire. ROMANDES. 